\title{
Characteristics and Breakdown Behaviors of Polysilicon Resistors for High Voltage Applications
}

\author{
Xiao-Yu Tang ${ }^{1}$ and Ke Dong ${ }^{2}$ \\ ${ }^{1}$ School of Electronic Science and Engineering, Nanjing University, Nanjing 210093, China \\ ${ }^{2}$ Shanghai HuaHong NEC Electronics Company, Shanghai 201206, China \\ Correspondence should be addressed to Xiao-Yu Tang; tangxiaoyu@smail.nju.edu.cn
}

Received 14 June 2014; Accepted 25 August 2014

Academic Editor: Rui Zhang

Copyright (c) $2015 \mathrm{X}$.-Y. Tang and K. Dong. This is an open access article distributed under the Creative Commons Attribution License, which permits unrestricted use, distribution, and reproduction in any medium, provided the original work is properly cited.

With the rapid development of the power integrated circuit technology, polysilicon resistors have been widely used not only in traditional CMOS circuits, but also in the high voltage applications. However, there have been few detailed reports about the polysilicon resistors' characteristics, like voltage and temperature coefficients and breakdown behaviors which are critical parameters of high voltage applications. In this study, we experimentally find that the resistance of the polysilicon resistor with a relatively low doping concentration shows negative voltage and temperature coefficients, while that of the polysilicon resistor with a high doping concentration has positive voltage and temperature coefficients. Moreover, from the experimental results of breakdown voltages of the polysilicon resistors, it could be deduced that the breakdown of polysilicon resistors is thermally rather than electrically induced. We also proposed to add an $\mathrm{N}$-type well underneath the oxide to increase the breakdown voltage in the vertical direction when the substrate is P-type doped.

\section{Introduction}

With the booming market of power integrated circuits for the smart power management $[1-4]$ and automotive and green energy, BCD $[5,6]$ (Bipolar-CMOS-DMOS) technology has been proved to be the best solution for these applications. People tend to integrate as much active and passive devices, including resistors, for different voltages' applications as possible on single chip. As a resistor, polysilicon [7] is supposed to be the best choice for integrated circuits technology due to its good and stable voltage and temperature coefficients. However, in the high voltage application, although the polysilicon resistor is still acting as an important device, there are few detailed reports about its properties, especially the voltage and temperature coefficients, under high voltages. It has been experimentally observed that different doping concentrations in the polysilicon could lead to different voltage and temperature coefficients of the resistor [8-10]. However, there is no enough physical explanation or further analysis for this phenomenon, which is very important for guiding the mass production. On the other hand, circuits for DC-DC and AC-DC applications in the smart power management system require the resistor to behave robustly under high voltages. This topic is not experimentally studied yet.

In this study, we investigate the voltage and temperature coefficients of polysilicon resistors with different doping concentrations. Secondly, the breakdown voltages $\left(V_{\mathrm{BD}}\right)$ of the resistors with different sizes are discussed. Also, the impacts of the high and low voltage stresses on the resistance of the polysilicon are experimentally studied. The results show that the voltage and temperature coefficients strongly depend on the doping concentration. After the high voltage stress, the resistance of the polysilicon could be degraded.

\section{Experimental}

The polysilicon resistors with different doping concentrations were prepared at $1 \mu \mathrm{m} \mathrm{BCD}$ standard process platform. The doping concentrations and corresponding square resistances are summarized in Table 1. Except for the doping concentration, all other processes were the same for all the resistors. The test structure for measurements in this study is shown in Figure 1. 
TABLE 1: Doping concentrations and corresponding square resistances at room temperature.

\begin{tabular}{lccc}
\hline & High & Medium & Low \\
\hline N-type doping concentration $\left(\mathrm{cm}^{-3}\right)$ & $2 E 20$ & $1.9 E 19$ & $1.5 E 19$ \\
Resistance at room temperature $(\mathrm{ohm} / \mathrm{sq})$ & $350 \pm 70$ & $2000 \pm 400$ & $10000 \pm 4000$ \\
\hline
\end{tabular}

$::$

(a)

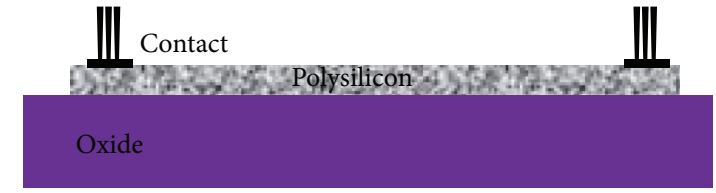

(b)

FIGURE 1: (a) Layout and (b) cross section of the resistor (color online).

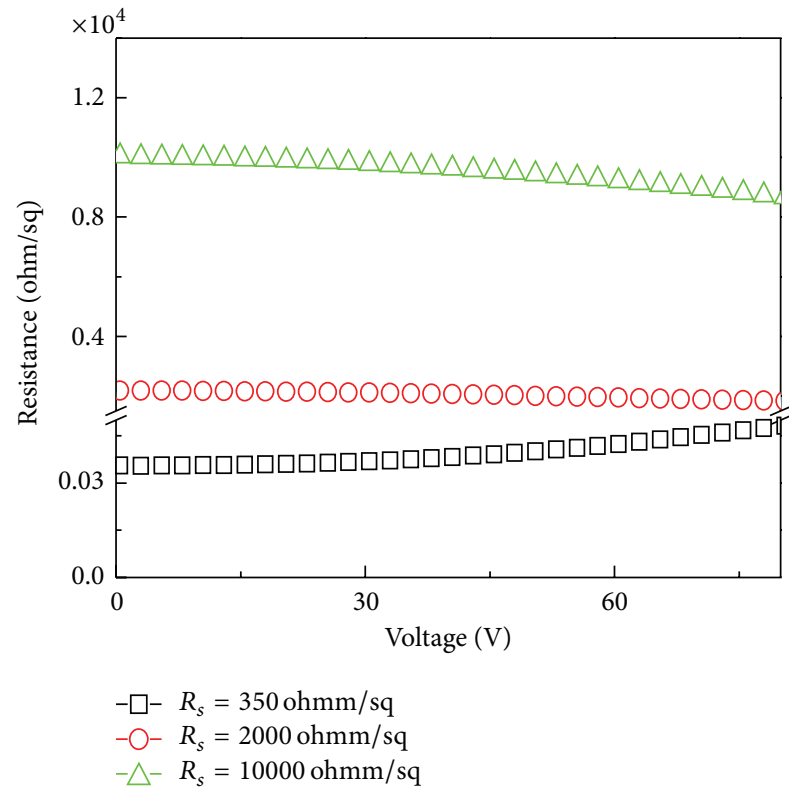

(a)

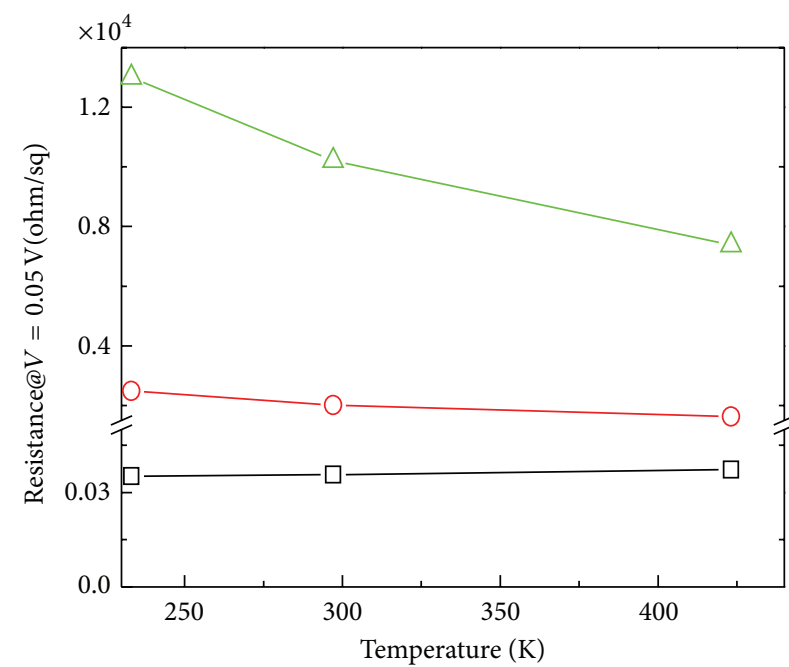

$-\square-R_{s}=350 \mathrm{ohmm} / \mathrm{sq}$

$-\mathrm{O}-R_{s}=2000 \mathrm{ohmm} / \mathrm{sq}$

$-\triangle-R_{s}=10000 \mathrm{ohmm} / \mathrm{sq}$

(b)

FIGURE 2: (a) Resistance-voltage and (b) resistance-temperature curves of three resistors with different resistances (color online).

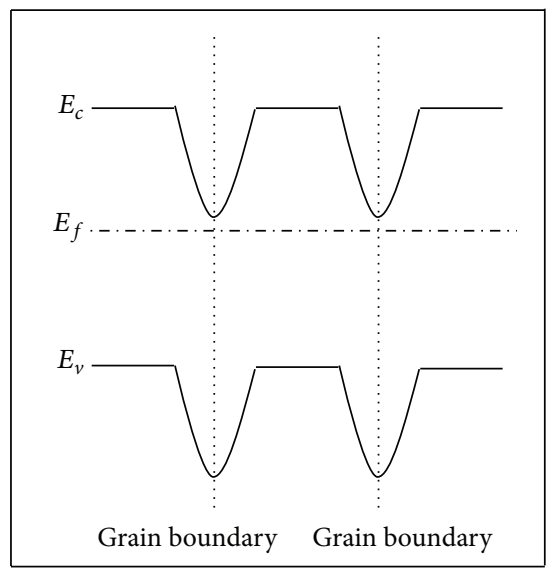

(a) Low doping

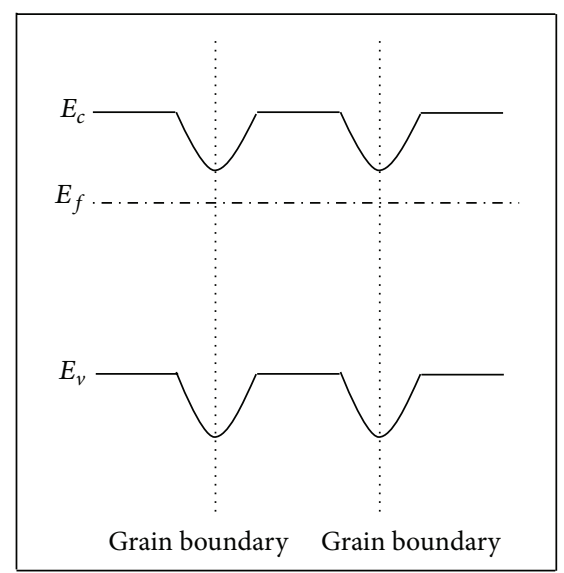

(b) High doping

FIGURE 3: Illustration of the energy band of (a) low doping and (b) high doping concentrations. 

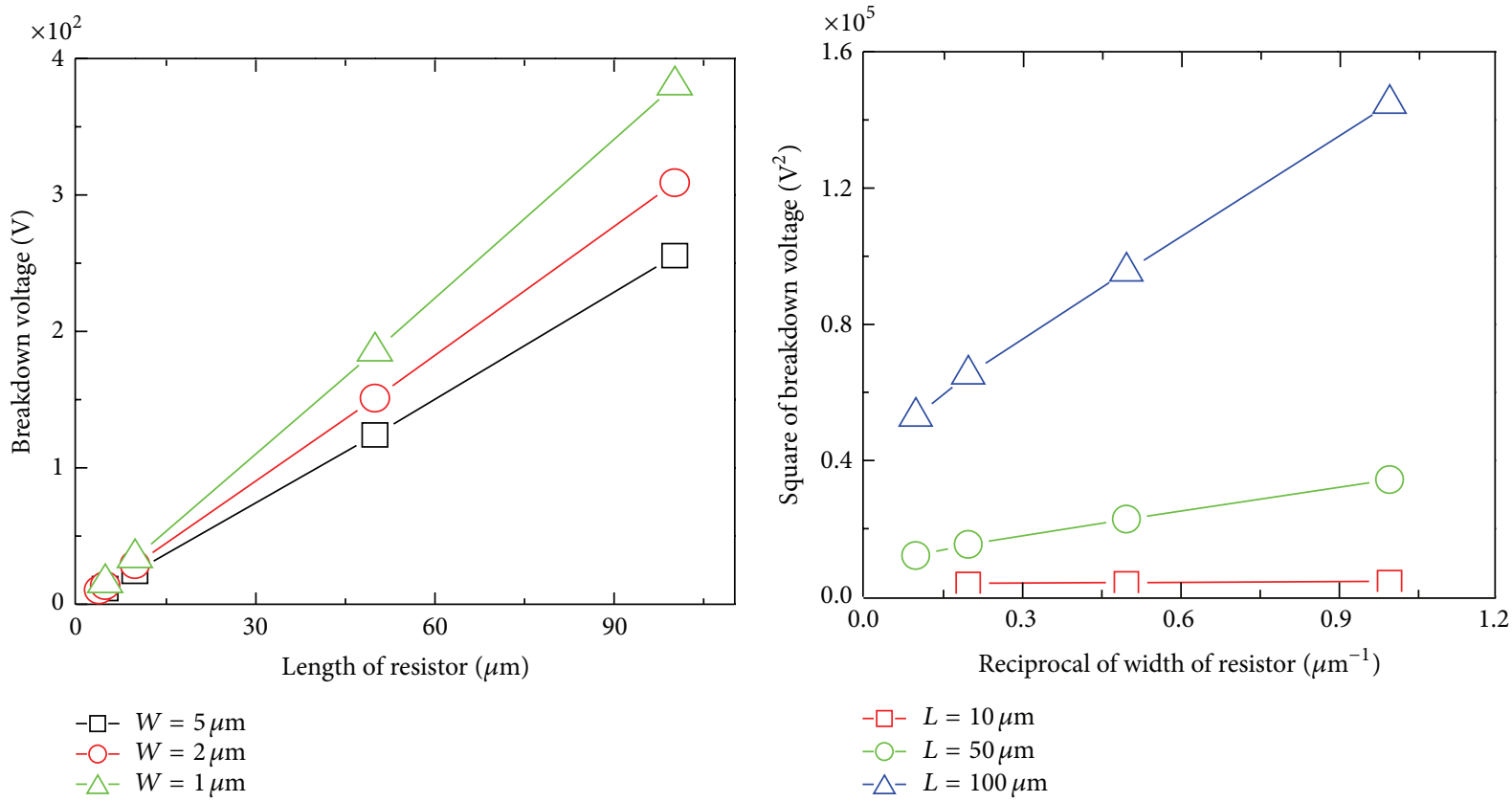

(a) Low doping

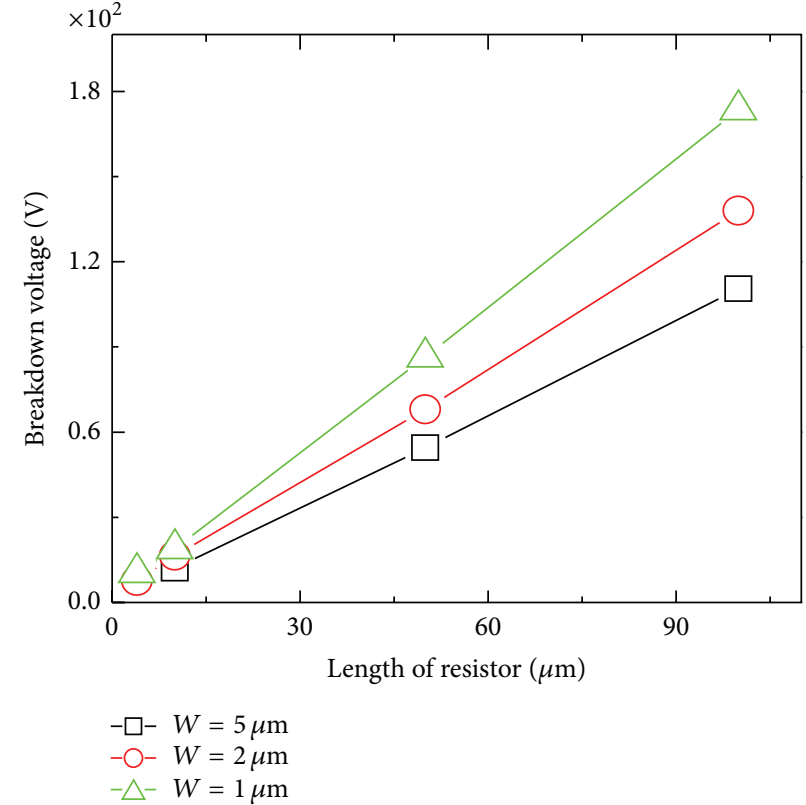

(c) High doping (b) Low doping

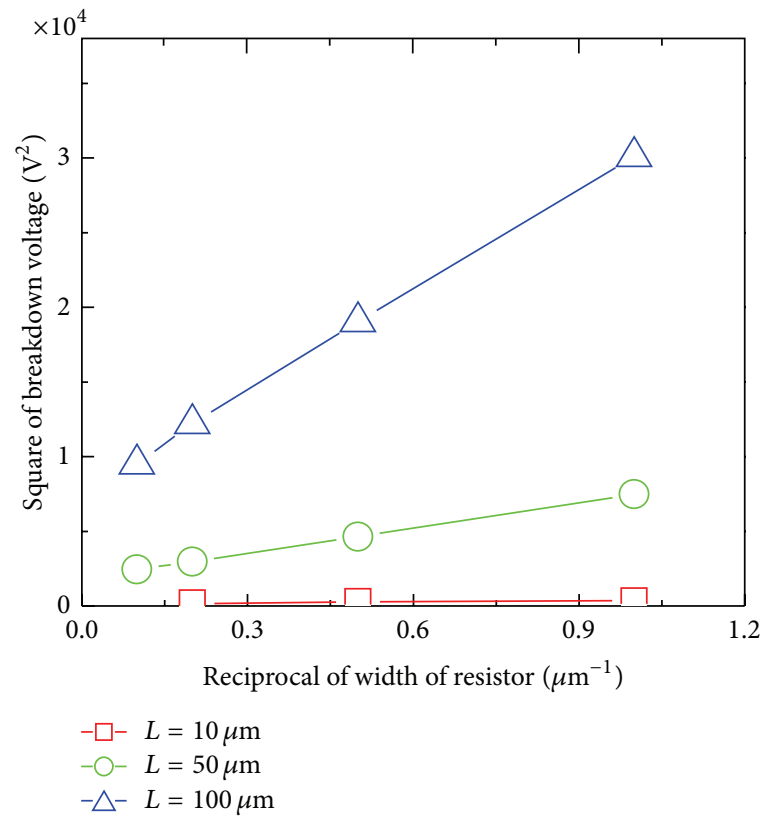

(d) High doping

FIGURE 4: $V_{\mathrm{BD}}$ versus length of the resistor $\left((\mathrm{a})\right.$ and (c)) and square of $V_{\mathrm{BD}}$ versus the inversed width of the resistor ((b) and (d)) (color online). (a) and (b) come from lightly doped resistors while (c) and (d) come from highly doped ones.

The voltage and temperature coefficients were extracted in the high voltage $(<80 \mathrm{~V})$ region and under the temperature varied from $-40^{\circ} \mathrm{C}$ to $150^{\circ} \mathrm{C}$.

$V_{\mathrm{BD}}$ of the polysilicon resistors with different widths $(W)$ and lengths $(L)$ were measured. Some polysilicon resistors were electrically stressed with a low voltage $(6 \mathrm{~V})$ or a high voltage $(80 \mathrm{~V})$ for some time.

\section{Results and Discussion}

3.1. Resistor Characteristics. Figure 2 shows the voltage and temperature coefficients of the polysilicon resistors with different doping concentrations. All the resistances in Figure 2(b) are obtained with applied voltage of $0.05 \mathrm{~V}$. It could be found from the figure that the resistor with a 
relative high doping concentration shows a positive voltage coefficient and a positive temperature coefficient while the other two resistors with much lower doping concentrations have negative voltage and temperature coefficients.

The possible reason for this phenomenon might be as follows. Polysilicon consists of many large grains, which are surrounded by grain boundaries. When the polysilicon is doped with a low impurity concentration, most of the carriers are absorbed by the grain boundaries, resulting in potential barriers around the boundary which could prevent the carriers moving (Figure 3). When a voltage is applied to the resistor, the carriers own a high energy and could more easily travel across the barrier, generating larger current and lower resistance. Similarly, a high temperature could help the carriers across the grain-boundary-induced barriers. Thus, the polysilicon resistor with a lower doping concentration has negative voltage and temperature coefficients. When the polysilicon is heavily doped, the grain boundaries have been full of constrained carriers while there are still many carriers remaining in grains. In this case, there will be little or even no grain-boundary-induced potential barriers around the boundary, while the high voltage and high temperature could cause large scattering within the crystalline grain, resulting in a larger resistance. Thus, the polysilicon resistor with a relative high doping concentration has positive voltage and temperature coefficients.

Therefore, it could be possible for us to make the voltage and temperature coefficients as small as we want if we tune the doping concentration carefully.

3.2. Reliability Behaviors of Polysilicon Resistors. Breakdown voltages of polysilicon resistors with different sizes ( $W$ and $L$ ) are shown in Figure 4. We can see that the breakdown voltage is proportional to the length of the resistor, while the square of the breakdown voltage is reversely proportional to the width of the resistor. This result is in good agreement to Scafft's research [11] on thermal conductance of a rectangular resistance on an oxide film over a Si substrate. The breakdown voltage decreases with the increase of the width of the resistor in Figure 4, showing that the polysilicon resistor is thermally breakdown, instead of electrically breakdown.

Furthermore, to study the reliability behaviors of the polysilicon resistors under high voltage applications, the same resistors with high doping concentrations were electrically stressed at $6 \mathrm{~V}$ and $80 \mathrm{~V}$. As shown in Figure 5, the stress of $6 \mathrm{~V}$ has no significant effect on the resistance, while the $80 \mathrm{~V}$ stress leads to a quick decrease and then a smooth increase in the resistance of the resistor. We think that the resistance decrease is due to the charge release from the grain boundaries while the increase could be attributed to selfheating of the polysilicon.

The above results suggest that, in order to ensure that the resistor behaves robustly in the circuits, the study on the breakdown voltage of the resistor with different sizes is required. Besides, the voltage stress should be a necessary item for the reliability evaluation including both self-heating and electromigration effects of polysilicon resistors [12-16].

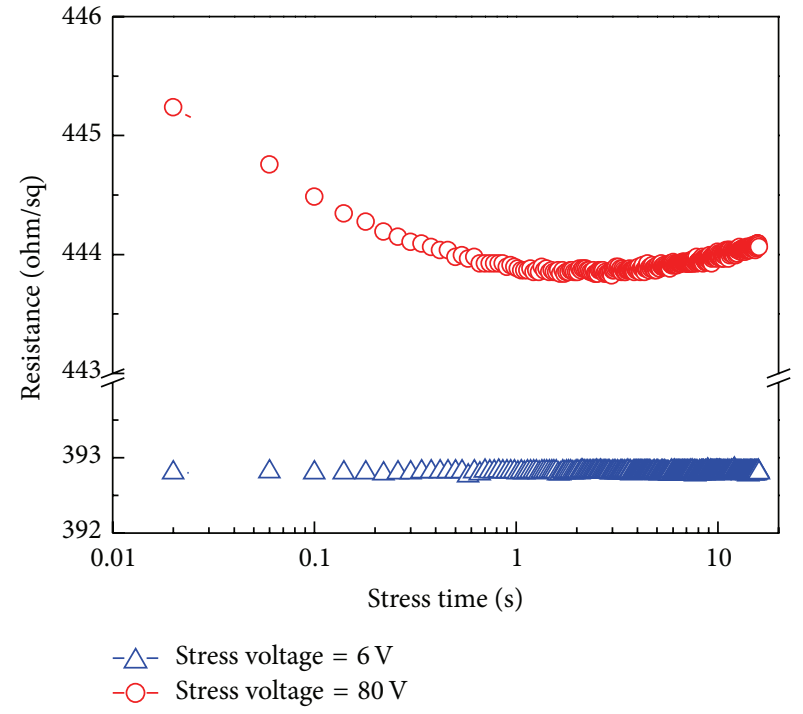

FIGURE 5: Normalized resistance under $6 \mathrm{~V}$ and $80 \mathrm{~V}$ stress (color online).

3.3. New Structure Proposal for Resistor due to Oxide Consideration. According to the structure of resistor in Figure 1, we can see that if the voltage application of the resistor comes up to several hundred volts, the voltage across the oxide is several hundred volts and the electrical field density is above the critical density of breakdown of silicon dioxide (Figure 6(a)). On the other hand, a high voltage on the resistor has a simultaneous vertical electrical field which may lead to a lot of reliability problems of the oxide for isolation. Thus, oxide breakdown becomes another shortcoming for high voltage application of resistor. A new structure in Figure 6(b) is suggested for the resistor to add a lightly doped N-type well under the oxide if the substrate is P-type doped. In this way, a PN diode is formed below the oxide. When high voltage is stressed on the resistor, the diode is reversed biased and undertakes parts of the voltage. The whole structure can be summarized as an oxide capacitance and a diode in series in Figure 6(c). We can see that the breakdown voltage of the vertical structure [11,12] can be increased and the voltage stressed on the resistor can be increased.

\section{Conclusions}

We have experimentally investigated the voltage and temperature coefficients of polysilicon resistors for high voltage applications. The experimental results show that ultralow voltage and temperature coefficients $(\sim 0)$ could be achieved according to carefully tuned doping concentrations. We also found that the breakdown of the polysilicon resistors under a high voltage is a thermal-dominant process, rather than an electrical process. Furthermore, the high voltage stress can degrade the performance of the polysilicon.

\section{Conflict of Interests}

The authors declare that there is no conflict of interests regarding the publication of this paper. 


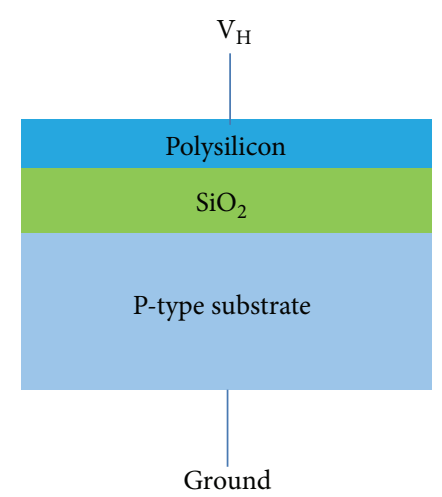

(a)

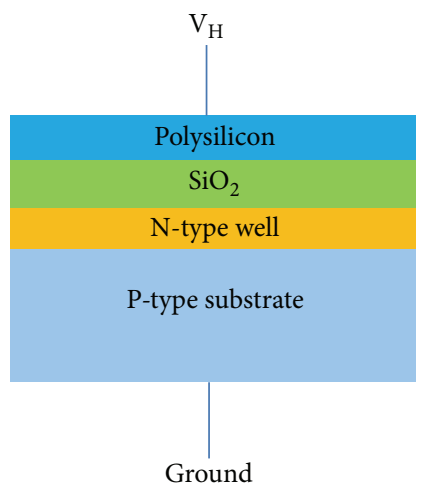

(b)

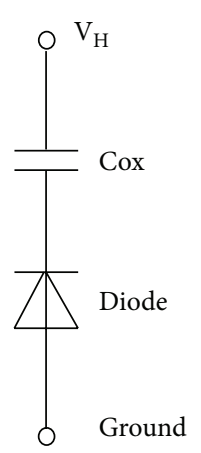

(c)
FIGURE 6: (color online) (a) Simplified vertical structure for resistor (b) added Nwell beneath the oxide to raise the breakdown voltage of the vertical structure.

\section{Acknowledgments}

The project was supported by the National Program on Key Basic Research Project (973 Program) of China (Grant no. 2011CBA00607), the National Natural Science Foundation of China (Grant nos. 61106089 and 61376097), Research Fund for the Doctoral Program of Higher Education of China (Grant no. 20130091110025) and BCD platform of Shanghai HuaHong NEC Electronics Company.

\section{References}

[1] R. Rudolf et al., IEEE International Symposium on Power Semiconductor Devices and ICs, 2011.

[2] K. Sam, N. A. Rahim, and H. Mokhlis, "Smart power management algorithm in microgrid consisting of photovoltaic, diesel, and battery storage plants considering variations in sunlight, temperature, and load," Energy Conversion and Management, vol. 84, pp. 562-582, 2014.

[3] T. Nitta, S. Yanagi, T. Igarashi et al., "Necessity of pulse hot carrier evaluation in suppressing self-heating effect for SOI smart power," in Proceedings of the 21st International Symposium on Power Semiconductor Devices and IC's (ISPSD '09), pp. 84-87, Barcelona, Spain, June 2009.

[4] D. Y. Zai, Y. Zhao, C. Yin-Fei et al., "Effect of the trench shape on the electrical properties of silicon based trench barrier schottky diode," Acta Physica Sinica, vol. 63, Article ID 127201, 2014.
[5] I.-Y. Park, Y.-K. Choi, K.-Y. Ko et al., "BD180-a new $0.18 \mu \mathrm{m}$ BCD (Bipolar-CMOS-DMOS) technology from 7V to 60V," in Proceedings of the 20th International Symposium on Power Semiconductor Devices and IC's (ISPSD '08), pp. 64-67, Orlando, Fla, USA, May 2008.

[6] A. Andreni, C. Claudio, and P. Galbiati, "A new integrated silicon gate technology combining bipolar linear, CMOS logic, and DMOS power parts," IEEE Transactions on Electron Devices, vol. 33, pp. 2025-2030, 1986.

[7] N. C.-C. Lu, L. Gerzberg, C.-Y. Lu, and J. D. Meindl, "Modeling and optimization of monolithic polycrystalline silicon resistors," IEEE Transactions on Electron Devices, vol. 28, p. 818, 1981.

[8] G. Baccarani, B. Riccò, and G. Spadini, "Transport properties of polycrystalline silicon films," Journal of Applied Physics, vol. 49, no. 11, pp. 5565-5570, 1978.

[9] J. Y. W. Seto, "The electrical properties of polycrystalline silicon films," Journal of Applied Physics, vol. 46, no. 12, pp. 5247-5254, 1975.

[10] P. J. French, "Polysilicon: a versatile material for microsystems," Sensors and Actuators A: Physical, vol. 99, no. 1-2, pp. 3-12, 2002.

[11] H. Scafft, "Thermal analysis of electromigration test structures," IEEE Transactions on Electron Devices, vol. 34, pp. 664-672, 1987.

[12] O. Semenov, A. Vassighi, and M. Sachdev, "Impact of selfheating effect on long-term reliability and performance degradation in CMOS circuits," IEEE Transactions on Device and Materials Reliability, vol. 6, no. 1, pp. 17-27, 2006.

[13] J. C. Doan, J. C. Bravman, P. A. Flinn, and T. N. Marieb, "The evolution of the resistance of aluminum interconnects during electromigration," Microelectronics Reliability, vol. 40, no. 6, pp. 981-990, 1999.

[14] L. He, L. Du, Y. Q. Zhuang et al., "A new model for electromigration grain boundary noise based on free volume," Chinese Physics B, vol. 19, Article ID 097202, 2010.

[15] H. Sheng-Dong, Z. Bo, L. Zhao-Ji, and L. Xiao-Rong, "A new structure and its analytical model for the vertical interface electric field of a partial-SOI high voltage device," Chinese Physics B, vol. 19, no. 3, Article ID 037303, 2010.

[16] Y. G. Wang, X. R. Luo, R. Ge et al., "Compound buried layer SOI high voltage device with a step buried oxide," Chinese Physics B, vol. 20, Article ID 077304, 2011. 

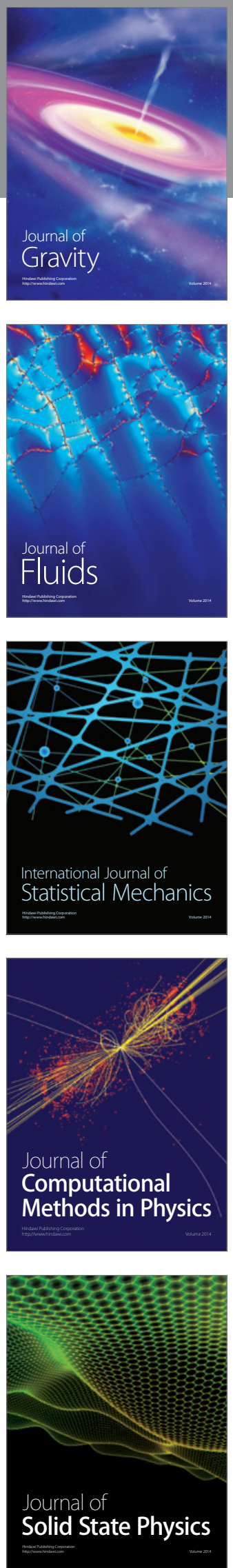

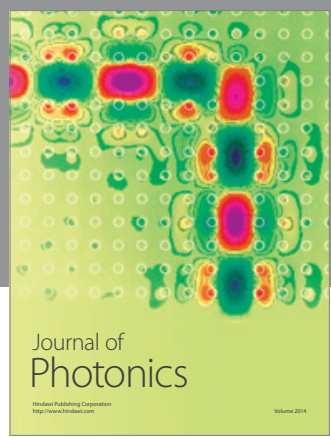

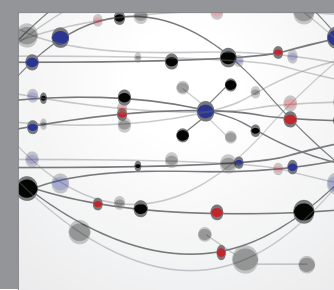

The Scientific World Journal

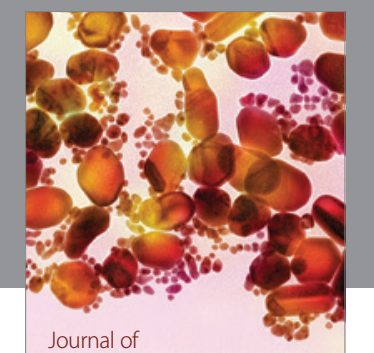

Soft Matter
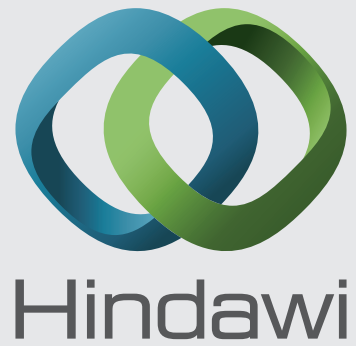

Submit your manuscripts at

http://www.hindawi.com
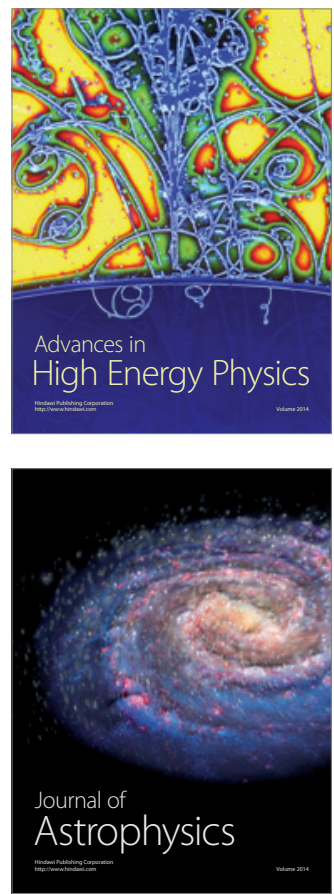
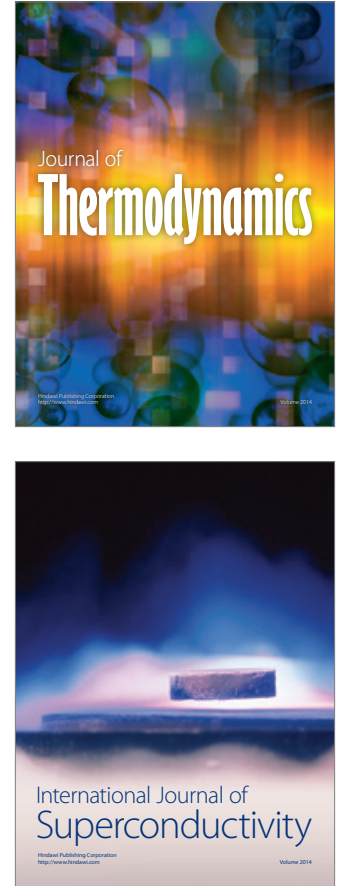
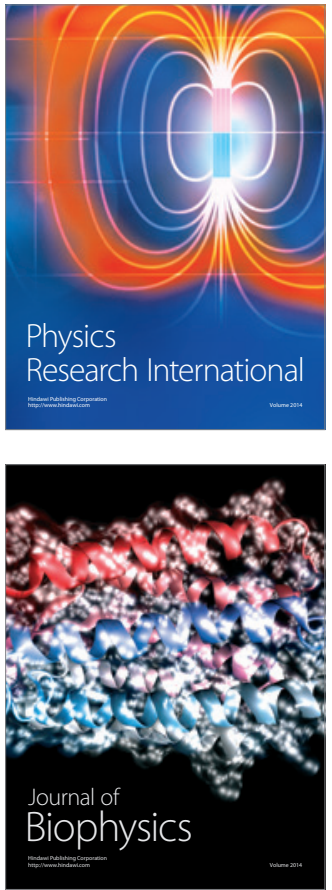
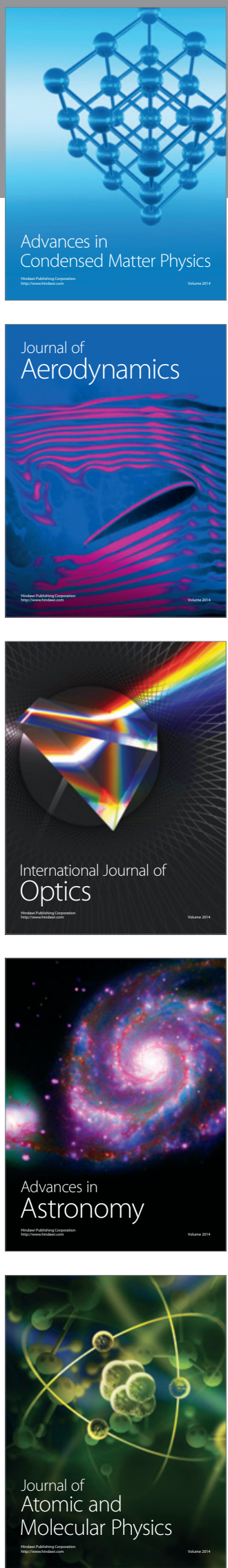